\title{
Entwicklung einer Anlage zur kontinuierlichen Herstellung von PET-Recyclingpolyolen
}

\author{
Rainer Langenstraßen, Stanislav Fulev, Andreas Apel, Bodo Gebert, Dieter Lehmann
}

\section{Einleitung}

Diese Arbeit hat die Entwicklung einer Anlage zum Ziel, mit der aus festem Polyethylenterephthalat (PET), wie es als Produktionsabfall bei seiner Herstellung und Verarbeitung entsteht, durch Umesterungen in Gegenwart von Glykolen und/oder oligomeren Estern Aromatische Polyesterpolyole (APP) als Rohstoffe für Polyurethane kontinuierlich hergestellt werden können. Die Arbeit baut auf den bisherigen Entwicklungen zur diskontinuierlichen Herstellung von Recyclingpolyolen aus PET-Abfällen an der Technischen Fachhochschule Wildau auf [1][2][3][4][5].

Mit Hilfe dieses Verfahrens können Polyesterpolyole verschiedener Eigenschaften für unterschiedliche Einsatzgebiete, z. B. Polyurethan-Hartschaumstoffe, -Beschichtungen, -Vergussmassen etc., hergestellt werden, die nur durch Modifizierungen in der Menge der Ausgangsstoffe und der Verfahrensbedingungen an die Erfordernisse angepasst werden. Dieses Verfahren liefert im diskontinuierlichen Betrieb qualitativ hochwertige Polyesteralkohole in einem breiten Parameterbereich. Auf Grund des hohen Aromatenanteils besitzen sie einen inhärenten Flammschutz [1][2].

Über Grundlagenuntersuchungen zum Lösevorgang von PET in verschiedenen Lösungsmitteln unter thermischer und mechanischer Beanspruchung und zur Umesterung der PET-Lösung zu oligomeren Produkten unter diskontinuierlichen Laborbedingungen, die auf einer Miniplantanlage ausgeführt wurden, ist bereits berichtet worden [4][5][6]. Aufbauend auf diesen Ergebnissen soll eine kontinuierlich arbeitende Anlage konstruiert und gebaut werden, die zur Bestimmung der Verfahrensparameter der kontinuierlichen Glykolyse von PET dient. Die in dieser Arbeit ermittelten verfahrenstechnischen und konstruktiven Grundlagen stellen die Basis zur Maßstabsvergrößerung für die Konstruktion und den Bau größerer Anlagen, einer Technikums- und darauf aufbauend einer Pilotanlage, dar.

Für ein kontinuierliches Verfahren zur Herstellung von Polyesterpolyolen aus PET gibt es weltweit kein Beispiel und auch keine geschützte Lösung, so dass mit dieser Arbeit wissenschaftliches und technisches Neuland betreten wird.

Das rohstoffliche Recycling der PET-Abfälle ist bisher nur diskontinuierlich durchgeführt worden [7][8][9][10]. Das diskontinuierliche Verfahren hat jedoch eine Reihe von Nachteilen, die vor allem in der langen Lösephase für das PET im Glykolyse-Gemisch, in längeren Reaktionszeiten, in den schwankenden Parametern der Produkte sowie in der zu geringen Wirtschaftlichkeit begründet liegen [11]. Ein kontinuierliches Verfahren hätte demgegenüber die Vorteile einer konstanten Produktqualität und einer verbesserten Wirtschaftlichkeit [12]. Nach eigenen Kostenabschätzungen gewinnt ein kontinuierliches Ver- fahren bei einem Durchsatz von mehr als etwa 1000 t/a an Wirtschaftlichkeit gegenüber einer diskontinuierlichen Verfahrensweise, da dann die Vorteile des kontinuierlichen Verfahrens (geringere Personalkosten, gleichbleibende Produktqualität) die Nachteile (höhere Investitionskosten, höherer Energiebedarf) überwiegen.

\section{Stand der Wissenschaft und Technik}

Eine Zusammenfassung zur Chemie der Herstellung von Polyethylenterephthalat einschließlich der Darstellung der nicht verwerteten Nebenprodukte und zur Glykolyse zu Polyesterpolyolen ist in der oben zitierten Arbeit [4] gegeben worden.

Die systematischen Untersuchungen sowohl des Lösevorgangs von PET in unterschiedlichen Lösegemischen als auch der daran unmittelbar anschließenden Umesterungsreaktion zur Herstellung von Polyesterpolyolen [4] ergaben ein Spektrum von optimalen Lösebedingungen insbesondere unter Verwendung von Diethylenglykol, gegebenenfalls im Gemisch mit anderen Glykolen, und dem bei der PET-Herstellung als Nebenprodukt anfallenden Oligoesterkondensat (OEK). Aus den Ergebnissen konnte für ein optimales Lösegemisch eine Zusammensetzung von 8 bis 13 Gew.-\% OEK in Diethylenglykol abgeleitet werden, das eine 3- bis 4-mal höhere Lösegeschwindigkeit für PET als reines Diethylenglykol (DEG) besitzt. Da die Lösegemische jedoch im Anschluss an den Lösevorgang als Glykolyse-Reagenzien dienen, ist die Entwicklung optimaler Löse- und Glykolysegemische unter Betrachtung sowohl der Löse- als auch der Glykolysephase einschließlich der Eigenschaften der erhaltenen APP vorzunehmen.

Wesentlichstes Ergebnis der Untersuchungen zur Umesterungsreaktion war die Ermittlung von Glykolysemischungen, die überraschenderweise sehr viel kürzere Reaktionszeiten ermöglichen, als bisher bekannt war. Die Reaktionszeiten betragen nur 12 bis 40 Minuten anstelle der sonst erforderlichen 4 bis 5 Stunden. Dieses Ergebnis ist für den kontinuierlichen Prozess von herausragender Bedeutung.

Ein wichtiger Kennwert der Polyole ist die Äquivalentkonzentration an Hydroxylgruppen (Hydroxylzahl, $\mathrm{OH}-\mathrm{Zahl}$, OHZ, Dimension mg KOH/g). Bei konstanter Funktionalität, d. h. der durchschnittlichen Anzahl Hydroxylgruppen je Molekül (bei den hier behandelten APP ist $f_{n}$ =2) steht diese in direktem Zusammenhang zur mittleren Molmasse und damit zu den Verarbeitungseigenschaften der Polyole und den Eigenschaften der daraus hergestellten Produkte. Um Polyesterpolyole mit definierten OH-Zahlen herzustellen, die zur Verarbeitung zu bestimmten Polyurethanen geeignet sind, muss die Umesterungsreaktion vor 
Erreichen des Reaktionsendes abgebrochen werden. Die Eignung der so erhaltenen Polyesterpolyole zur Herstellung von Polyurethan-Hartschaumstoffen wurde durch Formulierung zu A-Komponenten und deren Umsetzung mit dem Polyisocyanat Lupranat ${ }^{\circledR}$ M20A zu Hartschaumstoffen nachgewiesen. Insbesondere sind Polyol-Rezepturen entwickelt worden, die zu tieftemperaturstabilen PUR-Hartschaumstoffen (Dimensionsstabilität von -190 bis $+190{ }^{\circ} \mathrm{C}$ ) umgesetzt werden können.

Das Verfahren der Glykolyse von PET sollte sich auf Grund der bisher untersuchten Prozessvariablen und der Erkenntnisse aus vorhergehenden Versuchen zur Umesterung im Labormaßstab in kontinuierlicher Betriebsweise ausführen lassen. Im Rahmen vorhergehender FuE-Aktivitäten zur Herstellung von Recyclatpolyolen durch Glykolyse/Aminolyse von Polyurethanen [13] (dort weitere Zitate) waren im Versuchstechnikum der List AG (Schweiz) Versuche mit einem kontinuierlich arbeitenden Mischkneter durchgeführt worden, die zu überraschend guten Ergebnissen und weiter verkürzten Reaktionszeiten bei Recycling-Verfahren führten. Da das Verfahren zur Herstellung von PET-Polyesterpolyolen trotz völlig unterschiedlicher chemischer Abläufe und Reaktionsgeschwindigkeiten sowie unterschiedlicher mechanischer Eigenschaften der Ausgangsstoffe ähnliche Verfahrensabläufe erfordert wie das Verfahren der Glykolyse/Aminolyse von Polyurethanen, war die Annahme gerechtfertigt, dass sich auf der Grundlage der bisherigen Erfahrungen und Verfahrensentwicklungen ein kontinuierliches Verfahren in einem dazu geeigneten Apparat durchführen lässt. Daher ist der für diese Untersuchungen an der Technischen Fachhochschule Wildau verwendete Mischkneter der Firma List AG (Bilder 1 und 2) auch für die Entwicklung im Rahmen der vorliegenden Arbeit als Basis für die Weiterentwicklung zu Reaktoren für das PolyesterpolyolVerfahren eingesetzt worden, bei dem die Reaktionszeiten stoffbedingt länger sind.

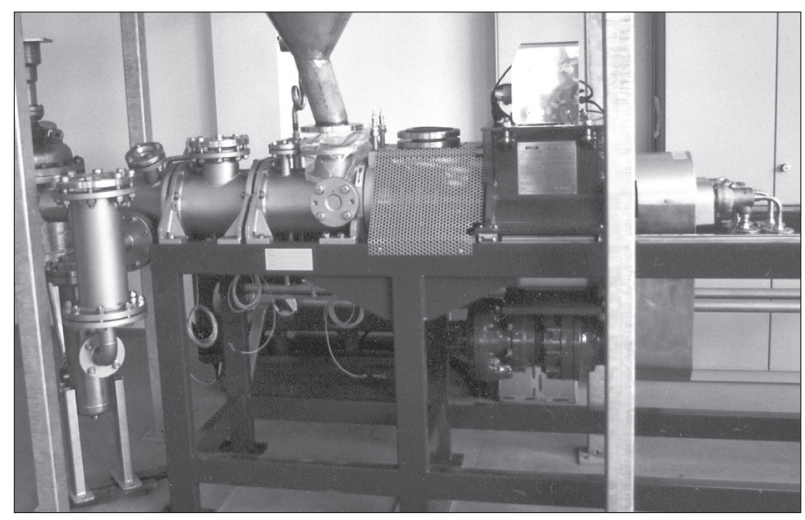

Bild 1: Zweiwellen-Mischkneter (Grundgerät)

Kontinuierlich arbeitende Knetreaktoren besitzen eine hervorragende Misch- und Knetwirkung für Mehrphasenreaktionen. Ihre Arbeitsweise ist dadurch charakterisiert, dass die Quervermischung des Reaktionsgutes von dessen axialem Transport weitgehend entkoppelt ist. Sie arbeiten umweltschonend und mit hoher Energieeffizienz und weisen eine präzise Produkttemperaturführung auf. Große freie Querschnitte garantieren eine effektive Abführung entstehender Gase und Dämpfe [14][15].

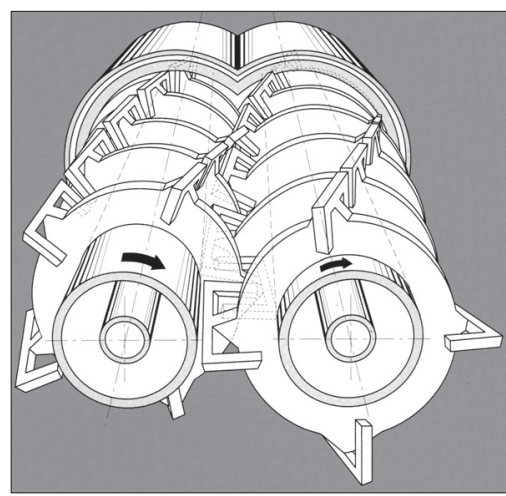

Bild 2: Zweiwellen-

Mischkneter

(Innenansicht)

Eine Patentrecherche zu kontinuierlich arbeitenden Reaktoren, die wie der an der Technischen Fachhochschule Wildau vorhandene Mischkneter nach dem Prinzip des Zweiwellenmischkneters aufgebaut sind, hat ergeben, dass derartige Geräte zwar im Prinzip bekannt sind, aber in aller Regel nicht für bestimmte Verfahren patentiert sind. Weiterhin sind wesentliche Merkmale, die für das zu untersuchende Glykolyse-Verfahren zur Herstellung von Polyesterpolyolen aus PET erforderlich sind, bei diesen Anlagen nicht vorhanden.

In der Recherche wurden Mischer, Mischkneter bzw. Reaktoren zur Durchführung von mechanischen, thermischen und/oder chemischen Prozessen mit flüssigen, hochviskosen oder rieselfähigen festen Stoffen ermittelt. Mit einer Ausnahme ist kein Bezug zu einem konkreten Stoffsystem genannt. Diese Geräte besitzen jeweils mehrere der folgenden Merkmale:

- zylindrisches oder (angenähert) doppelzylindrisches Gehäuse,

- (angenähert) waagerechte Anordnung,

- eine Welle oder (mindestens) zwei achsparallele Wellen,

- Wellen mit Rühr-/Knetelementen unterschiedlicher Gestaltung,

- Anordnung der Rühr-/Knetelemente mit Versatz,

- Rührwellen mit Schnecken,

- Gegenelemente an der Innenwand,

- die Anordnung der Rührelemente und Gegenelemente sorgt für eine Reinigung der Innenwand, der Rührelemente und der Gegenelemente,

- die Anordnung der Rührelemente und Gegenelemente bewirkt eine axiale Förderung des Produktes,

- durch die Rührelemente und Gegenelemente werden Knetspalten gebildet, die eine Knetung und Scherung des Produktes bewirken,

- Gehäuse beheizbar und/oder kühlbar,

- Wellen und/oder Rührelemente beheizbar und/oder kühlbar,

- absatzweise oder kontinuierliche Arbeitsweise ist möglich,

- in einem Fall ist ein Stator-Rotor-System mit Durchbrüchen genannt, durch die das Produkt periodisch durchtreten kann, wodurch es homogenisiert wird.

Die hier zu entwickelnde Anlage stellt entgegen den ermittelten technischen Lösungen eine speziell für die Durchführung der Glykolyse von Polyester-Reststoffen zur kontinuierlichen Herstellung von Polyesterpolyolen entwickeltes Gesamtsystem dar, das mit speziellen Merkmalen ausgestattet werden musste [16]. 


\section{Ergebnisse}

\subsection{Modellierung}

Die mathematische Modellierung der Löse- und Umesterungsschritte mit allen Nebeneffekten und -reaktionen ist die wesentliche Voraussetzung für die Konstruktion des Reaktors und die Simulation des darin ablaufenden Verfahrens. Die umfassende Kenntnis aller Prozessvariablen und deren Einfluss auf das Reaktionsgeschehen einschließlich der Temperaturverhältnisse und der Verteilungen im Reaktor ist die Voraussetzung für die Konstruktion und den Bau eines kontinuierlichen Reaktors, die kontinuierliche Durchführung des Verfahrens, eine weitgehende computergestützte Regelung und einen hohen Automatisierungsgrad des Gesamtverfahrens. Dazu sind folgende Teilaufgaben definiert worden:

- Entwicklung des Reaktorraumes der Miniplantanlage als Basis zur Entwicklung des kinetischen Reaktionsmodells,

- Entwicklung eines speziellen Rechenprogramms auf der Basis vorhandener Software zur Berechnung des Modells und

- weitergehende Verfeinerung des Modells zum Zweck der Beschreibung der verfahrenstechnischen Prozesse in der Miniplantanlage als Grundlage für den kontinuierlichen Reaktor.

Als Ergebnis dieses Komplexes wurde ausgehend von belastbaren Daten für das Verfahren zum Löseverhalten, zu den Reaktionsvariablen, der Zusammensetzung der Polyesterpolyole, der Reaktorgeometrie und den verfahrenstechnischen Variablen ein Modell für die Durchführung unter stationären sowie quasi-kontinuierlichen Bedingungen als Basis für die spätere Verfahrensdurchführung im kontinuierlichen Reaktor erwartet.

In der Literatur wurden mathematische Modellierungen der Depolymerisation von Nylon sowie des hydrolytischen Abbaus von PET ermittelt [17][18]. Die Methoden sind jedoch auf den vorliegenden Fall nicht anwendbar.

Da die chemische Reaktion mit ihren Abhängigkeiten von Temperatur, Druck, Mischungsvorgängen, Verweilzeiten usw. als wichtigste Komponente des Systems anzusehen ist, wurde die mathematische Modellierung zunächst auf den diskontinuierlichen Reaktionsablauf der Glykolyse beschränkt und mit einer Reihe von vereinfachenden Annahmen begonnen:

- homogene ortsunabhängige Reaktion

- Ausklammerung thermischer, mechanischer und katalytischer Einflüsse

- keine Berücksichtigung von Nebenreaktionen

- reines DEG als Glykolysereagens

Dazu wurde das Prinzip einer kinetischen Modellierung entwickelt und auf die Glykolyse von PET angewendet. Dabei wurde folgende Umesterungsreaktion betrachtet:

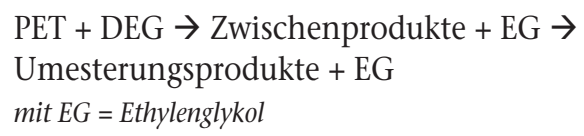

Bei dieser Reaktion werden die Kettenlängen von 75 bis 300 Einheiten beim PET auf $<10$ Einheiten bei den Produkten abgebaut. Zwei Modelle wurden entwickelt: a) das vollständige Modell und

b) das Klassenmodell.

Die Simulation nach a) bezieht alle Teilreaktionen der Umsetzung ein, indem sie alle vorkommenden Molekülkettenlängen einzeln betrachtet, während die Simulation nach b) nur Klassen von Reaktionen unterscheidet, indem jeweils 10 Kettenlängen des Ausgangsstoffes PET bzw. der Zwischenprodukte zusammengefasst sind. Dadurch sinkt die Zahl der Unbekannten auf ein beherrschbares Maß, und die Behandlung der gesamten PET-Umsetzungen wird möglich (s. Bilder 3 und 4).

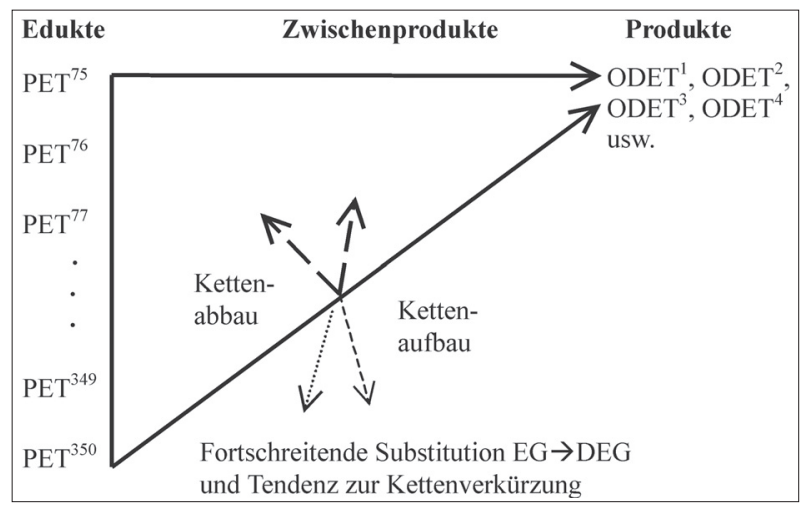

Bild 3: Schema der Hauptreaktion als vollständiges Modell

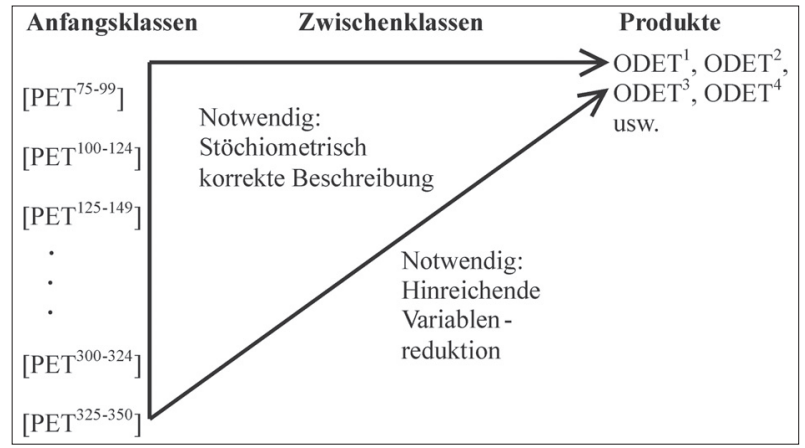

Bild 4: Schema für ein Klassenmodell

Das vollständige Modell wurde anschließend dem Klassenmodell gegenübergestellt. In diesem theoretischen Vergleich konnte das Klassenmodell dadurch bestätigt werden, dass hinreichend identische, plausible Kurvenverläufe für die Konzentrationen der Zwischen- und Endprodukte erhalten wurden. Die nachstehende Reaktionsgleichung drückt die PET-Degradation vereinfacht aus:

$$
\begin{aligned}
& \mathrm{PET}+\mathrm{DEG} \leftrightarrows \mathrm{ODET}+\mathrm{EG}(\uparrow) \\
& \text { mit ODET }={ }_{\text {,Oligodiethylenterephthalat" (Oligoester) }}
\end{aligned}
$$

Die Reaktion umfasst folgende Prozesse:

- Kettenverkürzung: PET $^{i}$, mit $i=75$... 350, geht über in ODET $^{\mathrm{j}}$, mit $\mathrm{j}=1$... 10 (i bzw. j: Anzahl der Monomereinheiten im Molekül).

- Substitution: EG wird in den Ketten durch DEG ersetzt.

- Komplexe Reaktionsdynamik: Neben endständiger Kettenverkürzung findet auch Kettenzerfall und Kettenwachstum statt.

Der theoretische Vergleich des Klassenmodells mit dem vollständigen Modell, zunächst am Beispiel sehr kleiner Polyesterketten, hat folgendes ergeben (s. Bild 5): 


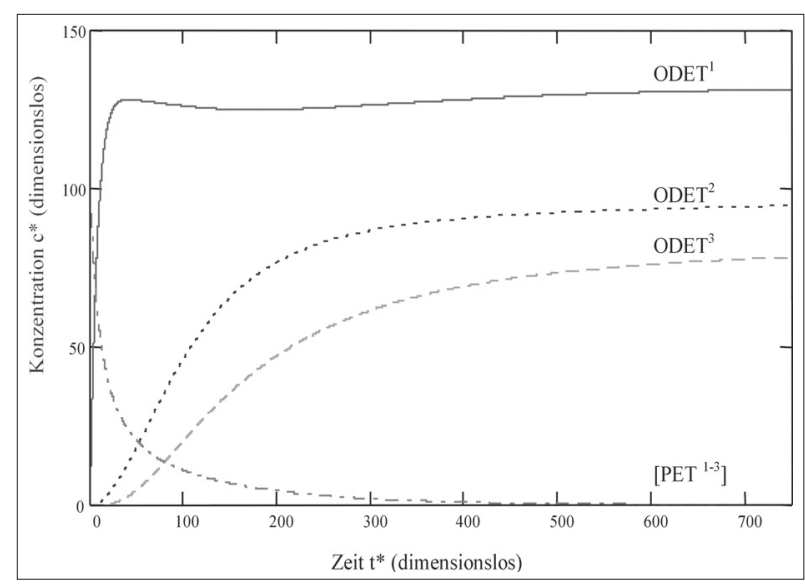

Bild 5: Darstellung eines Klassenmodells: Reaktionsverlauf von PET und ODET

- Im Falle der Umesterung von PET ${ }^{1}, \mathrm{PET}^{2}$ und $\mathrm{PET}^{3}$ ist es noch praktikabel, alle Substitutionsreaktionen zu behandeln. Daraus resultiert ein vollständiges Modell, welches man dem Klassenmodell gegenüberstellen kann. In diesem theoretischen Vergleich konnte das Klassenmodell bestätigt werden.

- Klassenvariablen sind folglich legitime Mittel der kinetischen Beschreibung.

Die Aufgabe bestand darin, das Klassenmodell dem vollständigen Problem der Herstellung von PET-Recyclingpolyolen anzupassen. Vor einer Erweiterung des Klassenmodells auf das gesamte reale Reaktionssystem ist ein erster experimenteller Nachweis der Übereinstimmung des Modells mit dem realen Reaktionsverlauf versucht worden, um den empirischen Beleg zur hohen Güte der Simulation komplexer chemischer Abbauprozesse in Klassen zu erbringen. Mit der experimentellen Verifikation erschienen folgende Ziele realistisch: Prognose der Produktzusammensetzung im Prozess des chemischen Recyclings, Optimierung von Rezepturen und kontinuierlichen Verfahrensabläufen, Anwendung des Modells auf weitere Degradationsverfahren.

Zur Vereinfachung des komplexen Reaktionssystems wurde Oligoesterkondensat (als Gemisch von wenigen niedermolekularen Oligoestern) mit Diethylenglykol umgesetzt, und zwar einmal unter Rückfluss und einmal unter Abdestillieren des entstehenden Ethylenglykols. Beide Versuche haben ergeben, dass in diesem Fall keine Umsetzung der höhermolekularen Bestandteile durch Glykolyse zu niedermolekulareren Produkten stattfindet, sondern im Gegenteil der Anteil der höhermolekularen Oligoester zunimmt. Die Esterkondensation überwiegt hier gegenüber der Spaltung.

Somit lässt sich dieses Experiment nicht als vereinfachte PET-Glykolyse auffassen, und es ist nicht aussagefähig zur Bestätigung des Klassenmodells. Als mögliche Erklärung kann angenommen werden, dass in einer Glykolysereaktion, die von PET ausgeht, das Konzentrationsverhältnis von OH-Gruppen im DEG $\left(\mathrm{c}_{\mathrm{OH}(\mathrm{DEG})}\right)$ $\mathrm{zu}$ OH-Gruppen im Polyester $\left(\mathrm{c}_{\mathrm{OH}(\mathrm{PET})}\right)$ am Beginn der Reaktion etwa

$$
\mathrm{c}_{\mathrm{OH}(\mathrm{DEG})}: \mathrm{c}_{\mathrm{OH}(\mathrm{PET})}=375: 1
$$

beträgt. Das entsprechende Verhältnis in der Reaktionsmischung des vorliegenden Experiments mit OEK beträgt dagegen etwa

$$
\mathrm{c}_{\mathrm{OH}(\mathrm{DEG})}: \mathrm{c}_{\mathrm{OH}(\mathrm{OEK})}=6: 1 \text {. }
$$

Daher konkurrieren die OH-Gruppen des DEG und der im OEK enthaltenen unterschiedlichen Oligoester im vorliegenden Experiment viel stärker miteinander um die Umesterungsreaktion an den Estergruppen, als es bei Glykolyse des Polyesters der Fall ist, mit der Folge, dass die Kettenverkürzung durch DEG nicht mehr die vorherrschende Reaktion ist, sondern von Kettenverlängerungs-, Veresterungs- und Umbaureaktionen der Oligoester untereinander überlagert wird.

Eine Weiterführung der Untersuchungen zur Modellierung sollte über drei Stufen von Versuchen führen:

- Experimente zur Bestimmung der Geschwindigkeitskonstanten und der Reaktionsordnung ohne Berücksichtigung der Abdestillation von Ethylenglykol. Ergebnis dieses Experimentes sollten die gemessenen Konzentrationen aller beteiligten Stoffe in ihrem zeitlichen Verlauf sein.

- Experimente zur optimalen Einstellung der Korrekturparameter. Unter unverändert gehaltenen Bedingungen, jedoch unter Berücksichtigung der Abdestillation von EG, sollte eine Folge von Konzentrationswerten erhalten werden, um einen Ansatz zu entwickeln, in dem die Destillation realistisch simuliert wird. Danach sollte das Klassenmodell durch Nutzung des fixierten Destillationsterms angepasst und auf dieser Grundlage die Korrekturparameter optimiert werden.

- Experimente zur Bewertung des Klassenmodells unter Variation des Temperaturverlaufs und Veränderungen in der stofflichen Zusammensetzung. Das Klassenmodell erweist sich dann als erfolgreich, wenn die optimierten Korrekturparameter auch auf diese Fälle anwendbar bleiben.

In der Erwartung, dass sich auf dieser Grundlage Formulierungen und Verfahrensbedingungen optimieren und die Zusammensetzung der Produkte nachvollziehen lassen, wäre bei erfolgreichem Verlauf dieser Experimente die vollständige Reaktion mittels des Klassenmodells beschrieben worden, so dass die Verfahrenssteuerung mit Hilfe dieses Modells ermöglicht oder mindestens unterstützt worden wäre.

Die hier zusammengefasste Modellierung ist einschließlich aller mathematischen Ableitungen und Schlussfolgerungen komplett in einer internen Studie niedergelegt [19].

Die bis hierher erhaltenen Ergebnisse werden als ein vielversprechender Ansatz zur Beschreibung jeder Art chemischer Abbauprozesse angesehen, die es wert sind, bei sich bietender Gelegenheit fortgesetzt zu werden, da erwartet werden kann, dass das Modell die Zusammensetzung der Produkte liefert, Rezepturen bzw. Verfahrensbedingungen optimieren kann, die Verfahrenssteuerung übernehmen kann und auf andere Depolymerisationsreaktionen übertragbar ist und dass sich Möglichkeiten der Theoriebildung eröffnen. 


\subsection{Versuchsanlage zur kontinuierlichen Glykolyse von PET}

\subsubsection{Konstruktion und Bau der Versuchsanlage}

Ziel dieses Arbeitsabschnitts ist ein funktionsfähiger, gegenüber dem im Aufbereitungstechnikum der Technischen Fachhochschule Wildau vorhandenen Mischkneter der List AG modifizierter und mit der erforderlichen Peripherie zu einer Versuchsanlage komplettierter Reaktor, in dem die anschließenden grundlegenden Untersuchungen zur kontinuierlichen Glykolyse von PET durchgeführt werden können.

Der vorhandene Mischkneter (s. Bilder 1 und 2) besteht aus einem mit Thermalöl beheizten Mantel und zwei beheizten Wellen, die hydraulisch angetrieben werden. Die Wellen arbeiten ineinandergreifend und gleichläufig mit einem Drehzahlverhältnis von 4:5. Auf den Wellen sind Knet- und Mischelemente derart angeordnet, dass mit ihrer Hilfe sehr hohe Scherkräfte erzeugt und durch diese eine sehr gute Zerkleinerung und Durchmischung des Reaktionsgemisches erreicht wird. Dabei ist die intensive Quervermischung von der axialen Transportbewegung weitgehend entkoppelt, so dass nur eine geringe Rückvermischung auftritt [14]. Die Reaktionstemperatur wird durch die ständige Kontrolle über das Thermalöl-Heizaggregat und eine Isolation der Reaktoraußenwand in sehr engen Grenzen konstant gehalten, um eine hohe Qualität des Reaktionsprodukts sicherzustellen.

Der anfängliche, im Projektantrag niedergelegte Plan sah folgenden Aufbau des Reaktors vor: Der Reaktor soll in einer ersten Zone, der Lösezone, Elemente zum schnellen Lösen von Feststoffen in einem erwärmten Glykolysegemisch aufweisen. Dazu sind die Glykolysereagenzien ständig vorgewärmt in diese Zone zu transportieren. Aus dieser Zone wird das Reaktionsgemisch in die zweite Reaktionszone transportiert, in der die eigentliche Umsetzung unter intensivem Umwälzen zur Beschleunigung der Transportprozesse erfolgt. Während der Umsetzung frei werdende flüchtige Stoffe werden am Ende der Zone abgezogen. In der dritten Zone erfolgt der Austrag über ein Filter, durch das ungelöste Anteile zurückgehalten werden. Nach dem Filtrieren des Reaktionsprodukts kann dieses in einen Sammeltank abgefüllt werden. Das Basisgerät soll zur Durchführung des kontinuierlichen Verfahrens durch folgende Entwicklungen gemäß den Anforderungen des kontinuierlichen Betriebs verändert werden:

- Ableitung von konstruktiven Elementen aus dem Reaktionsmodell sowie den verfahrenstechnischen Anforderungen für den Mantel und die Wellengestaltung,

- Entwicklung einer Konstruktion für den Innenraum mit einer Löse-, einer Umesterungs- und einer Nachbehandlungszone,

- Entwicklung und Konstruktion der Dosiereinrichtungen, insbesondere der Feststoffdosierung (PET- bzw. Mischpolymer-Granulate, -Flocken oder -Mahlgut),

- Entwicklung der Filtrations- und Entgasungseinheiten innerhalb oder außerhalb des Reaktors zum Entfernen fester und leichtflüchtiger bzw. gasförmiger Nebenprodukte,

- Einbauten zur Erfassung zusätzlicher Messdaten, z. B. eine Reihe von Widerstandsthermometern zur Erfassung des Temperaturverlaufs in den Reaktorzonen, in-line-Viskosimetersonden zur Ermittlung der Qualität der Durchmischung und Erfassung des Reaktionsfortschritts,

- Einbau einer Sonde am Reaktorausgang zur ständigen Kontrolle wichtiger Parameter, z. B. des OH-Gehaltes über FTIR-Messungen,

- Einrichtung zur Steuerung und Überwachung des Reaktors.

Im Verlauf der Bearbeitung haben sich auf der Basis der Grundlagenuntersuchungen [4], aus Diskussionen mit Fachleuten der Verfahrenstechnik, des Anlagenbaus und des Sondermaschinenbaus sowie bei Experimenten mit dem Reaktor während der Bau- und Erprobungsphase wesentliche Modifizierungen gegenüber dem anfänglichen Konzept erforderlich gemacht, um mit dem vorhandenen Grundgerät in kontinuierlichem Betrieb die Glykolyse von PET ausführen zu können. Die anfangs vorgesehene Arbeitsaufgabe, die Konstruktion und Bau von austauschbaren Rührelementen auf den Wellen und von Schikanen für die Reaktorwandung betraf, wurde nach den Ergebnissen aus den Versuchen sowohl an der Miniplantanlage als auch insbesondere am Mischkneter und nach den oben genannten Gesprächen nicht mehr als sinnvoll angesehen.

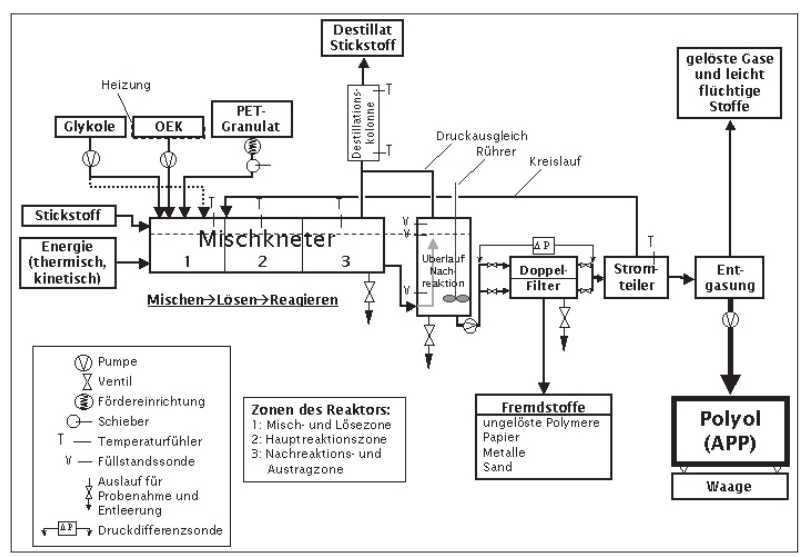

Bild 6: Fließschema der kontinuierlichen Glykolyseanlage

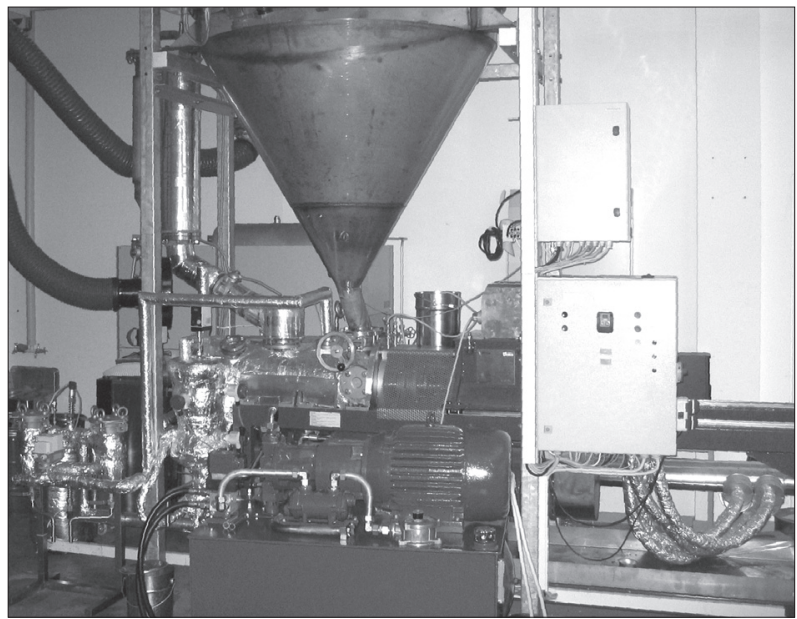

Bild 7: Gesamtansicht der kontinuierlichen Glykolyseanlage

Es wurde ein Anlagenkonzept mit teilweiser Kreislaufführung entwickelt. Bild 6 zeigt das Fließschema der konzipierten Anlage, Bild 7 eine Gesamtansicht der fertiggestellten Anlage. Die einzelnen Anlagen-Komponenten haben folgende Funktion: 
- Vorratsgefäße mit Dosierpumpen: Diese dienen der Zuführung der flüssigen Komponenten (Glykole, Katalysator-Lösungen) in den Reaktor.

- beheizbares Vorratsgefäß: In diesem Gefäß wird die OEK-Paste aufgeschmolzen, um sie anschließend mit einer Pumpe in den Reaktor fördern zu können.

- Rohrförderer für PET-Granulat: Eine Schnecke fördert das Granulat mit einstellbarer und steuerbarer Geschwindigkeit durch ein zylindrisches Rohr in den Reaktor.

- Pendelgasleitung (Druckausgleichsleitung): Diese sorgt für einen Druckausgleich, indem sie die Gasräume im Reaktor und im Überlaufbehälter miteinander und durch die Destillationskolonne mit der Atmosphäre verbindet und auf diesem Wege einen ausgeglichenen, dem Atmosphärendruck nahekommenden Druck in der Anlage sicherstellt. So wird garantiert, dass die Füllstände im Reaktor und im Überlaufbehälter gleich sind, und Störungen im Produktfluss werden ausschlossen.

- Kreislaufführung des Produktstroms: Es ist ein in der chemischen Reaktionstechnik übliches Verfahren, den Produktstrom teilweise in den Reaktor zurückzuführen (Schlaufen- oder Kreislaufreaktor) [20][21][22]. Dadurch wird insbesondere bei sehr komplexen Reaktionen eine Vergrößerung der Leistung des Reaktors, eine Einengung des Verweilzeitspektrums, eine Erhöhung der Selektivität und/oder eine Verbesserung der Ausbeute erzielt. In Abhängigkeit vom Rückführungsverhältnis nähert sich das Verhalten des Reaktors dem eines ideal durchmischten Rührkesselreaktors an. Ziel dieser Maßnahme ist es im vorliegenden Fall, durch Einstellung des Rückstroms und der Verweilzeit der Reaktionsmischung die Produkteigenschaften im Hinblick auf das gewünschte Spektrum zu steuern.

- Destillationskolonne: Die Destillationskolonne stellt ein komplexes Rückflusssteuerungssystem, bestehend aus Demister, Kühler und Wärmetauscher, dar. Sie dient der Fraktionierung und Abtrennung der flüchtigen Nebenprodukte, insbesondere des bei der Umesterung des PET entstehenden und abzudestillierenden Ethylenglykols sowie des mit den PET-Produkten eingeschleppten Wassers, aus der Reaktionsmischung.

- Überlauf-/Nachreaktionsbehälter: Dieser Behälter ist Bestandteil der Kreislaufführung. Der Inhalt des Behälters wird zur Beförderung der Nachreaktion durch einen Rührer vermischt. Der Behälter hat für Probenahmen ein Bodenablassventil.

- Füllstandsmesser: Da der Überlaufbehälter zur Füllstandssicherung im Reaktor und zur Nachreaktion der Reaktionsmischung dienen soll, muss der Füllstand durch Füllstandsmesssonden erfasst werden. Dadurch wird eine Steuerung wahlweise per Hand oder über das Prozessleitsystem möglich.

- Filter: Die Filteranlage ist als Doppelfilter ausgeführt, bei dem zwischen den beiden Filtern umgeschaltet werden kann. Auf der Basis einer Druckdifferenzmessung vor und hinter dem jeweils arbeitenden Filter wird der Zeitpunkt festgelegt, wann auf das andere Filter umgeschaltet und das erste Filter gereinigt wird. So ist eine Filtration im kontinuierlichen Betrieb möglich.

- Stromteiler: Der Stromteiler dient zur gezielten Einstellung der Teilung des Volumenstroms im Kreislaufsys- tem. Auf der Basis dieser Einstellung ist das Verhältnis zwischen Kreislaufstrom und Produktstrom bekannt.

- Entgasungseinrichtung: Auf Grund der kräftigen Vermischung des Reaktionsgutes im zu etwa zwei Dritteln gefüllten Reaktor enthält das Produkt gelöste Gase und leicht flüchtige Komponenten. Diese stellen bei der Herstellung von Polyurethanen aus dem Polyol einen unzulässigen Störfaktor dar, da sie bei der PolyurethanReaktion in Form von kleinen Gasblasen freigesetzt werden bzw. als Gasbildungskeime fungieren können, die infolge der fortschreitenden Härtung nicht mehr aus dem Material austreten und so zu Materialfehlern führen können. Aufgabe der Entgasungsanlage ist es, die gelösten Gase und insbesondere entstehende leicht flüchtige Komponenten in einer kontinuierlichen Arbeitsweise weitestgehend aus dem Polyol zu entfernen.

- Wärmeisolierung: Zur Minimierung von Wärmeverlusten des Heizsystems sind der Reaktor und alle Anlagenkomponenten einschließlich der Verbindungs- und Heizungsleitungen mit einer verkleideten Isolierschicht versehen.

- Schutzgasspülung: Zur weitgehenden Unterdrückung von Oxidationen, die zu Abbauprodukten und somit zu Qualitätsverminderungen, z. B. Verfärbungen, führen, ist eine Spülung der Gasatmosphäre mit einem Inertgas, z. B. Stickstoff, erforderlich.

- Steuerungssystem: Eine MSR-Technik und Steuersoftware, bestehend aus einer speicherprogrammierbaren Steuerung (SPS) und einem Prozessleitsystem, ermöglichen die Steuerung und Visualisierung der Anlage mit einem Computer. Die Heizeinrichtungen, die Pumpen und anderen Fördereinrichtungen und Ventile zur Steuerung der Stoffströme, der Rührer und die Messsonden für Temperaturen, Füllstände und Druckdifferenz sind mit dem Steuerungssystem verbunden. Das Steuerungssystem erlaubt sowohl eine Handsteuerung als auch den automatischen Betrieb der Anlage.

Die im Vorhaben anfangs vorgesehene IR-Sonde zur kontinuierlichen Bestimmung der OH-Zahl des Produktes wurde nicht verwirklicht, da es nach Erfahrungen aller Nutzer dieser Methode und nach eigenen Versuchen nicht möglich ist, auf diese Weise verlässliche Werte der OH-Zahl der Polyole zu erhalten. Das Gleiche gilt für NMR-Sonden. Die einzige Methode, die zuverlässige Werte für die $\mathrm{OH}$ Zahl liefert, ist die Titration nach Veresterung mit Acetanhydrid bzw. Phthalsäureanhydrid. Diese Bestimmung ist jedoch für den kontinuierlichen Betrieb zu zeitraubend. Eine schnelle Überprüfung der Produktqualität ist z. B. durch Messung der Viskosität von temperierten Proben (Viskosimeter Rheostress ${ }^{\circledR}$ 300, ThermoHaake) im Rotations- und Oszillationsmodus möglich. Eine direkte Messung der Viskosität im Produktstrom wäre wegen der hohen Temperatur und der daraus resultierenden sehr niedrigen Viskositätswerte mit einem entsprechend hohen Fehlerpotential dagegen nicht praktikabel.

\subsubsection{Betrieb der Versuchsanlage}

Die Erprobung der kontinuierlichen Anlage wurde auf der Basis von APP-Rezepturen, wie sie in den Grundlagenuntersuchungen an der Miniplantanlage entwickelt wurden, insbesondere derjenigen mit sehr kurzer Reaktionsdauer [4][23], ausgeführt. Dabei wurden die Ziele verfolgt, 
- diese kurzen Reaktionszeiten auf der kontinuierlichen Anlage zu bestätigen,

- zu untersuchen, welchen Einfluss das Verhältnis zwischen Kreislaufgeschwindigkeit und Gesamtströmung (sog. Rückführungsverhältnis) auf die Produkteigenschaften hat,

- Standardrezepturen zu entwickeln und

- mit auf der Anlage hergestellten APP Probeverschäumungen zu Polyurethan-Hartschaumstoffen zur Bestätigung der Anwendbarkeit auszuführen.

\section{a) Ermittlung der optimalen Verweilzeit}

Zunächst wurden Versuche mit vollständiger Kreislaufführung ausgeführt, um die optimale Verweilzeit im Reaktor für die Glykolyse von PET zu bestimmen. Die vollständige Kreislaufführung ohne kontinuierliche Produktentnahme ist als Batch-Versuch im kontinuierlichen Reaktor aufzufassen, die als Bindeglied zwischen echten Batch-Versuchen in der Miniplantanlage und kontinuierlicher Reaktionsführung im Mischknetreaktor dient.

Versuchsdurchführung: Die berechneten Mengen DEG und OEK werden aus den Vorratsgefäßen über die entsprechenden Pumpen bei laufenden Reaktorwellen in den vorgeheizten Reaktor einschließlich Überlaufbehälter eingefüllt. Der Reaktor wird auf $230^{\circ} \mathrm{C}$ geheizt. Die berechnete Menge PET wird auf einmal über den Rohrförderer zugegeben. Anschließend wird auf $250{ }^{\circ} \mathrm{C}$ geheizt. Die gesamte Reaktionsmischung wird vollständig im Kreislauf gefahren, d. h. das Rückführungsverhältnis beträgt $100 \%$. Während des Versuchs wird die Destillatmenge beobachtet. Nach beendetem Lösen des PET werden der Reaktionsmischung nach 15, 30, 45, 75 Minuten, 2 und 3 Stunden Reaktion Proben entnommen und der Versuch anschließend beendet. Von den Proben werden OH-Zahl, Säurezahl und Viskosität bestimmt. Als Glykolysemischung wurde ein Gemisch aus 81 Teilen DEG und 27 Teilen OEK auf 100 Teile PET verwendet. Der Lösevorgang wurde durch Probenahmen am Überlaufbehälter verfolgt. Er dauerte etwa 10 Minuten. Die anschließende Reaktionsphase ist in Bild 8 dargestellt, indem die Destillatmenge $\left(\mathrm{M}_{\text {Dest. }}\right)$ sowie die OH-Zahl und die Viskosität der entnommenen Proben über der Zeit aufgetragen sind. Nach den Ergebnissen ist bei der verwendeten Rezeptur je nach eingestellter Verweilzeit zwischen 30 und 75 Minuten eine OH-Zahl von 340 bis $280 \mathrm{mg} \mathrm{KOH} / \mathrm{g}$ erreichbar.

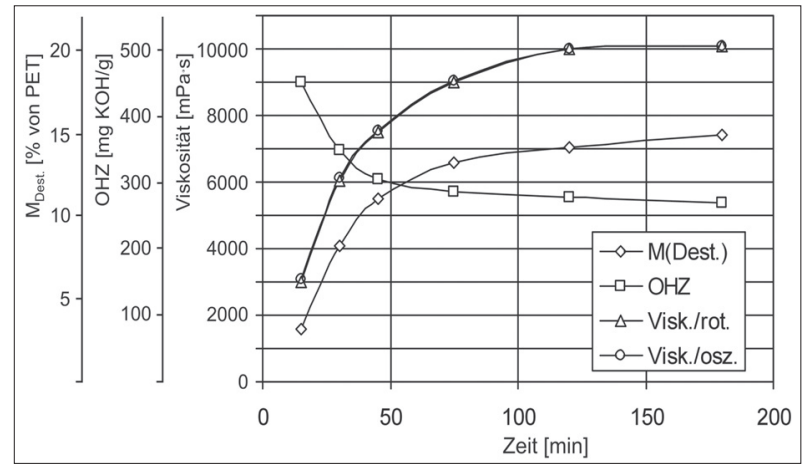

Bild 8: Verlauf der Glykolyse bei vollständiger Kreislaufführung

Auf Basis dieser Ergebnisse wurden kontinuierliche Versuche mit drei Verweilzeiten, die durch die Dosiergeschwindigkei- ten der Einsatzstoffe eingestellt wurden, im Bereich der oben ermittelten optimalen Verweilzeiten ausgeführt.

Versuchsdurchführung: Nach dem Befüllen des Reaktors wird anfangs wie oben auf 100\%igen Kreislauf und nach Ablauf einer Verweildauer (30, 45 bzw. 60 min, bezogen auf den Mischkneter) durch Umschalten des Stromteilers und Zuschalten der Dosiereinrichtungen und der Produktpumpe auf kontinuierlichen Betrieb (Durchlauf) umgeschaltet, wobei kein Kreislauf-Anteil gefahren wird (Rückführungsverhältnis 0 \%). Die Strömungsgeschwindigkeit wird jeweils entsprechend der Verweilzeit eingestellt. Dem Produktstrom werden halbstündlich Proben entnommen, von denen Analysen wie oben angefertigt werden. Die Ergebnisse bei drei verschiedenen Verweilzeiten sind in Bild 9 und 10 dargestellt.

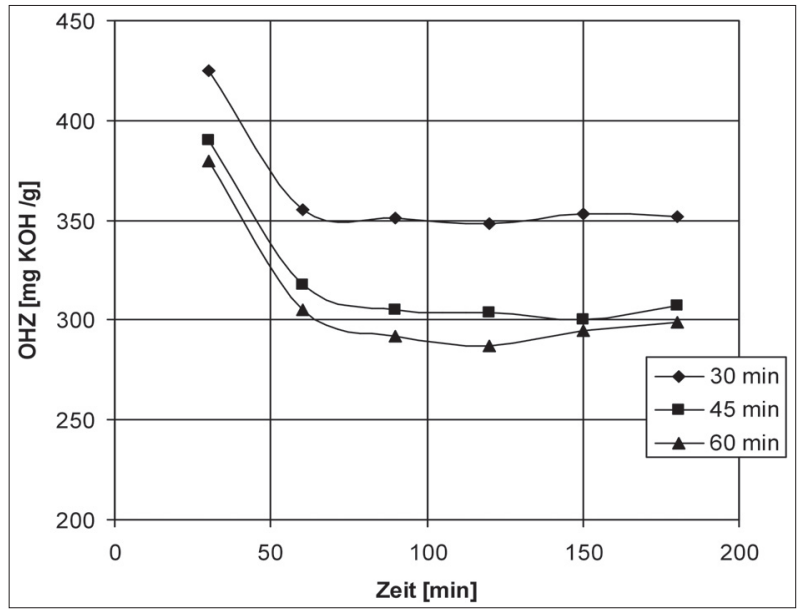

Bild 9: Verlauf der OH-Zahl der Reaktionsmischung bei unterschiedlichen Verweilzeiten

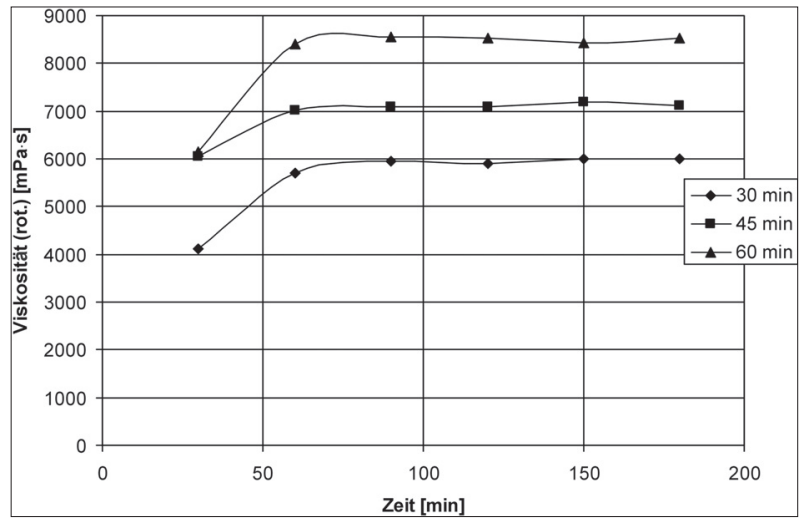

Bild 10: Verlauf der Viskosität der Reaktionsmischung bei unterschiedlichen Verweilzeiten

Die Verweilzeiten im Mischkneter entsprechen etwa folgenden Produktströmungsgeschwindigkeiten (Durchsatz):

\begin{tabular}{|l|l|}
\hline Verweilzeit & Durchsatz \\
\hline $30 \min$ & $24 \mathrm{l} / \mathrm{h}(26 \mathrm{~kg} / \mathrm{h})$ \\
\hline $45 \mathrm{~min}$ & $18 \mathrm{l} / \mathrm{h}(20 \mathrm{~kg} / \mathrm{h})$ \\
\hline $60 \mathrm{~min}$ & $12 \mathrm{l} / \mathrm{h}(13 \mathrm{~kg} / \mathrm{h})$ \\
\hline
\end{tabular}

Aus diesen Versuchen ergibt sich, dass nach einer relativ kurzen Einlaufzeit (Einlaufkurve etwa 30 Minuten) die Produktparameter im wesentlichen konstant sind und den Werten aus dem Batch-Versuch (s. Bild 8) entsprechen. Alle angeführten Verfahrensbedingungen haben 
sich bewährt und können entsprechend den jeweils angestrebten Parametern des Polyols eingestellt werden.

\section{b) Untersuchung des Einflusses des Kreislaufanteils}

Wie schon im Abschnitt 3.2.1 erwähnt, wurde die Glykolyseanlage als Schlaufen- bzw. Kreislaufreaktor ausgeführt, um das Verweilzeitspektrum einengen, die Selektivität und Leistungsfähigkeit des Reaktors erhöhen sowie die Produkteigenschaften gezielt beeinflussen zu können. Die Versuche hierzu wurden analog den kontinuierlichen Versuchen unter a) (zweite Vorschrift) ausgeführt, jedoch unter Einstellung verschiedener Kreislaufanteile, und zwar 20, 40, 60 und $80 \%$. Die Versuche unter a) entsprechen dem Kreislaufanteil $0 \%$. Die Verweilzeit wurde jeweils so eingestellt, dass sie 45 Minuten unter den Bedingungen ohne Kreislauf entspricht. Die Versuchsergebnisse sind in den Bildern 11 und 12 dargestellt.

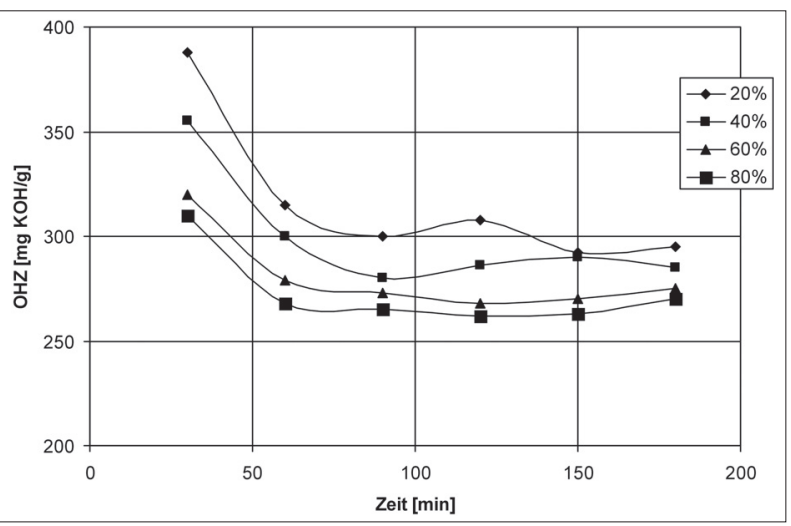

Bild 11: Verlauf der OH-Zahl der Reaktionsmischung bei unterschiedlichen Rückführungsverhältnissen

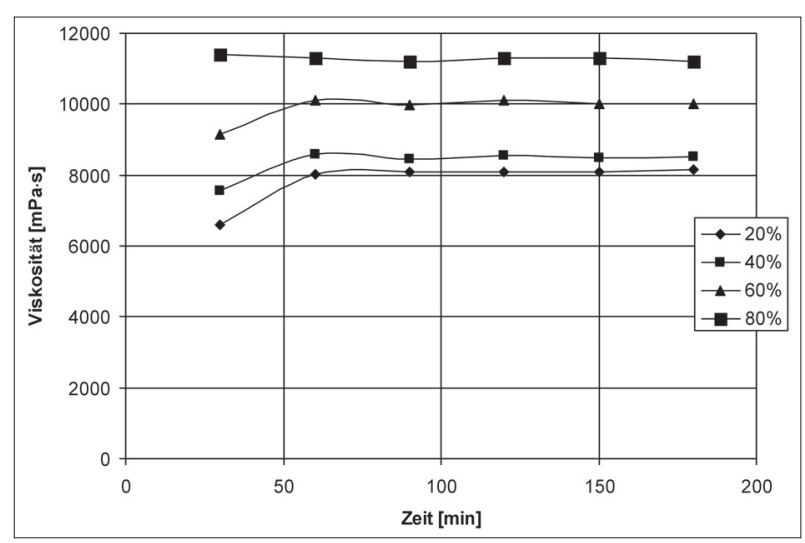

Bild 12: Verlauf der Viskosität der Reaktionsmischung bei unterschiedlichen Rückführungsverhältnissen

Nach kurzen Einlaufzeiten stellen sich bei den erprobten Rückführungsverhältnissen jeweils relativ konstante Produktparameter ein. Bei höheren Rückführungsverhältnissen werden Produktparameter wie bei längeren Verweilzeiten erhalten.

Nach den bisherigen Ergebnissen hat sich die Fahrweise mit teilweiser Rückführung bewährt. Es kann erwartet werden, dass die Größe des Rückführungsverhältnisses einen Einfluss auf die Stabilität der Produktparameter, insbesondere der Viskosität und der Kristallisationsneigung, hat, da das Verweilzeitspektrum verändert wird. Nach dem gegenwärtigen Entwicklungsstand scheint ein Rückführungsverhältnis von $60 \%$ optimal.

\section{c) Anpassung der Rezeptur}

Auf der Basis der unter a) und b) erprobten Reaktionsbedingungen wurden mehrere Rezepturen auf der Anlage in längeren Versuchsläufen erprobt. Proben zur Analyse der Produktparameter wurden dem Produktstrom halbstündlich entnommen. Tabelle 1 zeigt einige Ergebnisse.

\begin{tabular}{|c|c|c|c|c|c|}
\hline Versuchs-Nr. & 1 & 2 & 3 & 4 & 5 \\
\hline \multicolumn{6}{|c|}{ Rezeptur [Teile auf 100 T. PET]: } \\
\hline DEG & 81 & 81 & 81 & 60 & 76 \\
\hline PEG 200 & 0 & 0 & 10 & 0 & 0 \\
\hline Octandiol & 0 & 0 & 0 & 20 & 16 \\
\hline OEK & 27 & 27 & 26 & 20 & 26 \\
\hline \multicolumn{6}{|l|}{ Reaktionsbedingungen: } \\
\hline Verweilzeit [min] & 30 & 45 & 45 & 30 & 30 \\
\hline Rückführungsverhältnis [\%] & 0 & 40 & 80 & 60 & 40 \\
\hline Versuchsdauer [h] & 6 & 8 & 8 & 8 & 8 \\
\hline \multicolumn{6}{|l|}{ Produkteigenschaften: } \\
\hline $\mathrm{OH}-\mathrm{Zahl}[\mathrm{mg} \mathrm{KOH} / \mathrm{g}]$ & $350 \pm 4$ & $288 \pm 3$ & $281 \pm 4$ & $282 \pm 4$ & $310 \pm 5$ \\
\hline Säurezahl [mg KOH/g] & 1,0 & 0,9 & 0,96 & 0,8 & 1,1 \\
\hline$\eta_{\text {rot } / 25^{\circ} \mathrm{C}}[\mathrm{mPa} \cdot \mathrm{s}]$ & $\begin{array}{l}5900 \\
\pm 150 \\
\end{array}$ & \begin{tabular}{|l|}
8510 \\
\pm 120 \\
\end{tabular} & $\begin{array}{l}7950 \\
\pm 150 \\
\end{array}$ & \begin{tabular}{|l|}
9810 \\
\pm 180 \\
\end{tabular} & $\begin{array}{l}8020 \\
\pm 130 \\
\end{array}$ \\
\hline$\eta_{\text {osz } / 25^{\circ} \mathrm{C}}[\mathrm{mPa} \cdot \mathrm{s}]$ & $\begin{array}{l}5960 \\
\pm 140\end{array}$ & $\begin{array}{l}8560 \\
\pm 110\end{array}$ & $\begin{array}{l}8030 \\
\pm 170\end{array}$ & $\begin{array}{l}9860 \\
\pm 150\end{array}$ & $\begin{array}{l}8070 \\
\pm 150\end{array}$ \\
\hline
\end{tabular}

Tabelle 1: Kontinuierliche Versuche mit unterschiedlichen Rezepturen

Nach diesen Versuchen hat sich die kontinuierliche Glykolyseanlage im längeren Betrieb bewährt und liefert aromatische Polyesterpolyole in konstanter Qualität.

\section{d) Probeverschäumungen}

Aus den unter c) hergestellten APP-Mustern wurden PURHartschaumstoffe nach den Standardformulierungen in Tabelle 2 hergestellt. Tabelle 3 zeigt die Ergebnisse.

\begin{tabular}{|l|l|l|l|l|l|}
\hline Formulierung Nr. & $\mathbf{1}$ & $\mathbf{2}$ & $\mathbf{3}$ & $\mathbf{4}$ & $\mathbf{5}$ \\
\hline Zusammensetzung der A-Komponente (Mengen in \%): \\
\hline APP Nr. & 90 & 07 & 093 & 093 & 093 \\
\hline APP-Menge & 94 & 75 & 94 & 80 & 89 \\
\hline Katalysatorgemisch & 0,6 & 21 & 1 & 15 & 5 \\
\hline Tegostab $^{\circledR} 8433$ & 0,4 & - & 0,5 & - & \\
\hline Tegostab $^{\circledR} 8404$ & - & 1 & - & 1 & 1 \\
\hline Wasser & 5 & 3 & 4,5 & 4 & 5 \\
\hline B-Komponente (auf 100 \% A-Komponente): \\
\hline Lupranat ${ }^{\circledR}$ M20A & 134 & - & 125 & 408 & 306 \\
\hline Lupranat & \\
\hline
\end{tabular}

Tabelle 2: Beispielformulierungen für PUR-Hartschaumstoffe aus APP

\begin{tabular}{|c|c|c|c|c|c|}
\hline Formulierung $\mathrm{Nr}$. & 1 & 2 & 3 & 4 & 5 \\
\hline Raumgewicht [g/l] & 33 & 45 & 50 & 60 & 80 \\
\hline \multicolumn{6}{|c|}{ Dimensionsstabilität [\%]: } \\
\hline $120^{\circ} \mathrm{C}$ & $-1,4$ & $+0,3$ & 0 & $-0,8$ & $-0,3$ \\
\hline $150^{\circ} \mathrm{C}$ & $-2,7$ & $+3,5$ & 0 & $+0,2$ & $-2,5$ \\
\hline $170^{\circ} \mathrm{C}$ & $-3,9$ & - & $+0,7$ & $+0,8$ & $-3,8$ \\
\hline$-130^{\circ} \mathrm{C}$ & $-1,2$ & $-3,8 \%$ & - & $-0,2$ & $-1,0$ \\
\hline$-170^{\circ} \mathrm{C}$ & $-4,5$ & - & $-0,4$ & $-0,6$ & $-3,5$ \\
\hline$-190^{\circ} \mathrm{C}$ & - & - & $-0,8$ & $-2,5$ & - \\
\hline Druckfestigkeit [MPa] & 0,08 & 0,09 & 0,17 & 0,25 & 1,45 \\
\hline Biegefestigkeit [MPa] & - & 0,33 & 0,50 & 0,65 & - \\
\hline Bruchdehnung [\%] & - & 14,0 & 7,8 & 7,7 & - \\
\hline
\end{tabular}

Tabelle 3: PUR-Hartschaumstoffe aus in der kontinuierlichen Anlage hergestellten APP-Mustern 
Aus den in der kontinuierlichen Glykolyseanlage hergestellten APP sind PUR-Hartschaumstoffe mit dem gleichen Eigenschaftsspektrum herstellbar wie aus den bisher diskontinuierlich hergestellten APP [3].

\section{Zusammenfassung}

Aufbauend auf den Ergebnissen an der Miniplantanlage zur Glykolyse von PET [4] [23] wurde durch Ergänzung mit neuen Bauelementen gemäß den verfahrenstechnischen Anforderungen des kontinuierlichen Betriebs, die aus den Ergebnissen der Grundlagenuntersuchungen abgeleitet wurden, und durch Entwicklung eines Steuerungssystems eine kontinuierlich arbeitende Anlage entwickelt, konstruiert und gebaut. Mit dieser Anlage wurden Untersuchungen des Löse- und Umesterungsverhaltens der Polymeren in den Glykolysemitteln ausgeführt und so die Verfahrensparameter für die kontinuierliche Herstellung der APP festgelegt. Diese Parameter wurden durch Erprobung mit Standardmischungen zur Herstellung von APP einschließlich Charakterisierung der hergestellten Polyole und Probeverschäumungen bestätigt.

Die mathematische Modellierung der komplexen chemischen Reaktion führte zu einem vielversprechenden Ansatz zur Beschreibung chemischer Abbauprozesse.

Die ermittelten verfahrenstechnischen und konstruktiven Grundlagen stellen die Basis für die Konstruktion und den Bau größerer Anlagen nach Abschluss des Vorhabens zunächst bei einem internationalen Partner dar.

\section{Danksagung}

Das dieser Arbeit zugrunde liegende Vorhaben wurde mit Mitteln des Bundesministeriums für Bildung und Forschung unter dem Förderkennzeichen 03I0202 gefördert. Die Verantwortung für den Inhalt dieser Veröffentlichung liegt bei den Autoren. Die Autoren danken dem Bundesministerium für Bildung und Forschung für die finanzielle Unterstützung der hier dargestellten Arbeiten im Rahmen des InnoRegio-Wettbewerbs.

Den Firmen List AG, Arisdorf (Schweiz), CTA Anlagenbau GmbH, Fürstenwalde, TSA GmbH, Doberlug-Kirchhain, Lausitzer Edelstahltechnik GmbH, Doberlug-Kirchhain, und Sigmar Mothes Hochdrucktechnik GmbH, Berlin, sei für hilfreiche konstruktive Beratung, Entwicklung und Ausführung der Anlagenkonstruktion herzlich gedankt. Der Firma Krauss-Maffai Kunststofftechnik GmbH, München, danken wir für die kostenlose Bereitstellung von PET-Ware und der Firma Trevira GmbH, Guben, für die kostenlose Bereitstellung von Oligoesterkondensat. Herrn Karl-Heinz Schmidt danken wir für die Herstellung und Prüfung von Hartschaumstoff-Proben.

\section{Literatur}

[1] G. Behrendt, A. Lobeda, M. Pohl: Verfahren zur Herstellung von Polyesteralkoholen sowie Polyesteralkohole; DE-OS 19918650 (16. 4. 1999/27. 1. 2000); PCT/WO 99/54380 (16. 4. 1999/28. 10. 1999)

[2] G. Behrendt, M. Pohl, H.-D. Hunger: Polyester-Polymerpolyole für Polyurethane und Verfahren zu ihrer Herstellung; DE-PS 19915125 (25. 03. 1999/19. 10. 2000/5. 7. 2001)

[3] R. Eftimova, Y. Loseva, K.-H. Schmidt, M. Wotzka, P. Wagner, G. Behrendt: Polyester Polyols from Waste PET Bottles for Polyurethane Rigid Foams; Wissenschaftliche Beiträge der TFH Wildau 2003, S. 19-25

[4] R. Langenstraßen, S. Fulev, A. Apel, B. Gebert, D. Lehmann, G. Behrendt: Entwicklung der Grundlagen für eine Laboranlage zur kontinuierlichen Herstellung von PET-Recyclingpolyolen (Darstellung des Standes der Arbeiten im InnoRegio-Projekt); Wissenschaftliche Beiträge der TFH Wildau 2004, S. 34-45

[5] R. Langenstraßen: Kontinuierliche Herstellung von aromatischen Polyesterpolyolen aus PET-Abfällen, Projekt im Rahmen der InnoRegio-Initiative des BMBF; Poster auf dem Tag der offenen Tür der TFH Wildau 2004

[6] R. Langenstraßen, S. Fulev, R. Evtimova, G. Behrendt: Investigation of the Polyethylene Terephthalate (PET) Transesterification Reaction; Vortrag auf der Ninth Conference of Chemistry and Physical Chemistry of Oligomers, Odessa, Sept. 2005

[7] Ch.-H. Chen et al.: Studies of Glycolysis of Poly(ethylene terephthalate) Recycled from Postconsumer Soft-Drink Bottles. I.; Journal of Applied Polymer Science, 80 (2001), S. 943-948; II., loc. cit., S. 965-962; III., loc. cit. 87 (2003), S. 2004-2010

[8] J. Milgrom: Polyethylene Terephthalate; in R. J. Ehrig (Hrsg.): Plastics Recycling, Products and Processes; Carl Hanser Verlag, München Wien New York Barcelona 1992

[9] F. P. La Mantia, M. Vinci: Recycling poly(ethylene terephthalate); Polymer Degradation and Stability 45 (1994), S. $121-125$

[10] T. Spychaj, D. Paszun: New Trends in Chemical Recycling of Poly(ethylene terephthalate); Macromol. Symp. 135 (1998), S. $137-145$

[11] M.-D. Umbach: Marktstudie: Aromatische Polyesterpolyole (APP); Diplomarbeit, TFH Wildau 2003

[12] G. Kielburger: Von Batch zu Konti; Process (2003) 1, S. 3

[13] S. Fulev, R. Langenstraßen, G. Behrendt: Untersuchungen zur Herstellung von aromatischen Polyesterpolyolen für elastische Polyurethane; Wissenschaftliche Beiträge der TFH Wildau 2005, S. 74-78

[14] J. List: Großvolumige Knetreaktoren; cav 5/94, Nr. 288; J. List: Kontinuierlicher Misch-/Knetreaktor; ibid. 09/2003, S. 42, Nr. 445; LIST-CRP, Firmenschrift List AG, 2000

[15] A. Diener: Herstellen und Aufarbeiten von Polymeren mit großvolumigen Knetern; Vortrag auf dem 6. Kunststoffkolloquium, Schwarzheide, 15. 9. 2005

[16] G. Behrendt, R. Langenstraßen, B. Gebert, H.-D. Hunger, A. Diener, Th. Isenschmid: Verfahren und Vorrichtung zur Herstellung von Recyclat-Polyolen aus Polyestern; DE-OS 102004014163 (17. 3. 2003/9. 12. 2004)

[17] A. Ogale: Depolymerization of Nylon 6: Some Kinetic Modelling Aspects; Journal of Appl. Polym. Science 29 (1984), S. 3947-3954 
[18] J. R. Campanelli, M. R. Kamal, D. G. Cooper: A Kinetic Study of the Hydrolytic Degradation of Polyethylene Terephthalate at High Temperatures; Journal of Appl. Polym. Science 48 (1993), S. 443-451

[19] A. Apel: Mathematische Modellierung der Umesterung von PET zu aromatischen Polyesterpolyolen; unveröffentlichte Studie, Technische Fachhochschule Wildau, 2004

[20] H. Franke, K. Schiefer (Hrsg.): Lueger Lexikon der Verfahrenstechnik, Stuttgart 1970

[21] K. Winnacker, L. Küchler: Chemische Technik, Prozesse und Produkte, 5. Aufl., Weinheim

[22] M. Baerns, H. Hofmann, A. Renken: Chemische Reaktionstechnik, Stuttgart, New York 1987

[23] R. Langenstraßen, A. Apel, S. Fulev, B. Gebert, D. Lehmann: InnoRegio FIRM: Entwicklung der Grundlagen für eine Anlage zur kontinuierlichen Herstellung von PET-RecyclingPolyolen; Schlussbericht, Wildau 2006

\section{Autoren}

\section{Dr. Rainer Langenstraßen}

Technische Fachhochschule Wildau

Fachbereich Ingenieurwesen/Wirtschaftsingenieurwesen Tel. +49 3375 508-502

rainer.langenstrassen@tfh-wildau.de

Dipl.-Math. Andreas Apel

Technische Fachhochschule Wildau

Fachbereich Ingenieurwesen/Wirtschaftsingenieurwesen

Dipl.-Ing. Stanislav Fulev

Technische Fachhochschule Wildau

Fachbereich Ingenieurwesen/Wirtschaftsingenieurwesen

Dipl.-Ing. Bodo Gebert

Technische Fachhochschule Wildau

Fachbereich Ingenieurwesen/Wirtschaftsingenieurwesen

Dipl.-Ing. Dieter Lehmann

Technische Fachhochschule Wildau

Fachbereich Ingenieurwesen/Wirtschaftsingenieurwesen 